\title{
Perfil nutricional e alimentar de portadores de HIV-1/AIDS internados em um hospital universitário
}

\author{
Nutritional and alimentary profile of HIV-1/AIDS patients admitted in a university hospital
}

\author{
Luanny Kaísa de Oliveira Kauffmanna, Rozinéia de Nazaré Alberto Mirandab, \\ Aldair da Silva Guterresc ${ }^{c}$, Amanda Fernandes Pinto ${ }^{a}$ \\ acadêmica de Nutrição. Universidade Federal do Pará/UFPA. Instituto de Ciências da Saúde. Belém, Pará, Brasil. \\ Nutricionista. Professora Doutora em Biologia de Agentes Infecciosos e Parasitários. Universidade Federal do Pará/UFPA. Instituto de Ciências da Saúde. Belém, Pará, Brasil. \\ c Nutricionista. Doutora em Biologia de Agentes Infecciosos e Parasitários. Hospital Universitário João de Barros Barreto (HJUBB/UFPA). Belém, Pará, Brasil.
}

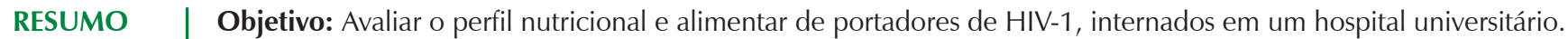
Materiais e Métodos: Tratou-se de um estudo transversal, descritivo, no qual a população de estudo foi composta por 54 pacientes internados, de ambos os sexos, adultos, com faixa etária de 20 a 59 anos, acompanhados durante o período de Abril a Outubro de 2014, na Clínica de Doenças Infecciosas e Parasitárias (DIP), do Hospital Universitário João de Barros Barreto (HUJBB/UFPA). Foi utilizado formulário próprio para o levantamento das características demográficas, socioeconômicas, antropométricas e dietéticas (QFA). Para verificar a as diferenças de médias foi realizada a análise de variância (ANOVA) e o grau de associação entre as variáveis foi medido pela correlação de Pearson, estabeleceu-se como significância valor de $p<0,05$.

Resultados: Prevalência do sexo masculino, os pacientes pertencem a um nível econômico baixo, apresentando também uma baixa escolaridade. O perfil nutricional encontrado nos pacientes, de acordo com os métodos de avaliação antropométrica (índice de massa corporal, prega cutânea tricipital, circunferência do braço e circunferência muscular do braço), apresentou predominância de desnutrição (55,5; 92,6; 79,6 e 55,5\%; respectivamente), evidenciando déficit de massa magra e gordura corporal. Encontrou-se ainda significativa correlação linear do índice de massa corpórea com as outras medidas antropométricas compartimentadas. Quanto à ingestão dietética, verificou-se expressivo consumo de alimentos energéticos, significativo consumo de alimentos construtores e baixa ingestão de alimentos reguladores quando comparados ao recomendado.

Conclusão: Observou-se que a maioria dos pacientes apresentou estado nutricional de desnutrição e o perfil alimentar, de modo geral, se caracterizou por prevalência de hábitos alimentares saudáveis.

Palavras-chave: HIV; antropometria; desnutrição; estado nutricional; consumo de alimentos.

ABSTRACT Objective: To assess the nutritional status and feeding habits of HIV-1 patients admitted to a university hospital.

Materials and Methods: This was a cross-sectional, descriptive study in which the studied population was composed by 54 hospitalized patients of both sexes, adults, aged from 20 to 59 years, assessed over the period from April to October 2014, at the Infectious and Parasitic Diseases Clinic (IPD), of the University Hospital João de Barros Barreto (HUJBB/UFPA). A specific form to survey the demographic, socioeconomic, anthropometric and dietary (FFQ) characteristics was used. The differences of means was verified by the analysis of variance (ANOVA) test and the level of association between variables was measured by Pearson's correlation test. The values were significant when $p<0.05$.

Results: Males were more prevalent and the patients presented low economic level situation and low level of education. The nutritional status found in patients according to the anthropometric assessment methods (body mass index, triceps skin fold, arm circumference and arm muscle circumference), presented prevalence of malnutrition (55.5; 92.6; 79.6 and 55.5\%; respectively), showing lean mass and body fat deficit. It was found also an important linear correlation between body mass index with the other compartmentalized anthropometric measurements. Regarding dietary intake, it was verified a significant consumption of energetic and builder foods and low intake of regulator foods when compared to the recommended intake values.

Conclusion: It was observed that most patients showed nutritional condition of malnourishment and the dietary profile was characterized, in general, by the prevalence of healthy eating habits.

Keywords: HIV; anthropometry; malnutrition; nutritional status; food consumption.

\section{Correspondência:}

LUANNy KaísA DE OLIVEIRA KAUfFMANN

Travessa Quinta linha, Conjunto Ipaupixuna

Rua Dois, Quadra D, no 11 - Tenoné

66820-160 Belém, PA, Brasil

E-mail: luannykaisa@gmail.com 


\section{INTRODUÇÃO}

A síndrome da imunodeficiência adquirida (SIDA, AIDS - Acquired Immunodeficiency Syndrome) é a manifestação clínica extrema da infecção por um retrovírus, o vírus da imunodeficiência humana (VIH, HIV - Human Immunodeficiency Virus) ${ }^{1}$. Ele tem a capacidade de inserir seu material genético em células alvo-hospedeiras, principalmente os linfócitos T CD4, os quais constituem células de defesa do sistema imunológico humano. Após a infecção e ampla replicação, o vírus destrói várias células e, posteriormente, infecta outras. Esta infecção é uma doença de espectro amplo, com curso clínico variável, progressiva supressão do sistema imunológico, indução a infecções oportunistas recorrentes, alterações nutricionais, debilitação progressiva e morte ${ }^{2}$.

Em 1980, no estado de São Paulo, foi diagnosticado o primeiro caso da doença no Brasil, e atualmente é uma epidemia de alcance mundial. As estimativas são de que aproximadamente 718 mil indivíduos vivam com o HIV/AIDS no Brasil, porém apenas $80 \%$ conhecem seu diagnóstico 3 .

O HIV pode originar um progressivo aparecimento de desnutrição devido a uma ingestão alimentar diminuída, ao aumento das necessidades energéticas, à alteração da absorção e do metabolismo dos nutrientes e à interação da terapia com os nutrientes. Esta patologia enfraquece o sistema imunitário, aumentando o risco de aparecimento de infeções oportunistas e mortalidade nestes doentes. São sinais e sintomas da desnutrição a perda de peso, perda de massa magra e massa gorda, déficit em micronutrientes (vitaminas e minerais) e competência imunitária diminuída ${ }^{4}$.

É fundamental uma avaliação nutricional criteriosa nesses indivíduos, investigando as deficiências nutricionais de forma global ou isolada, sendo necessário um estudo dos índices antropométricos e biológicos ${ }^{5}$. A manutenção do estado nutricional depende de uma combinação de fatores como ingestão apropriada de alimentos, absorção eficiente de nutrientes e metabolismo adequado ${ }^{6}$. O papel da nutrição é fundamental para otimizar o estado nutricional do doente através da avaliação, diagnóstico, intervenção e monitorização nutricional. O nutricionista deve fornecer ao doente uma terapia individualizada e educação a nível nutricional ${ }^{4}$.

Poucos são os estudos nacionais que avaliaram o estado nutricional de pacientes hospitalizados por HIV/AIDS, o que ressalta a necessidade de mais estudos que visem conhecer a frequência e os fatores que levam à desnutrição nesses pacientes. Essas informações são fundamentais para que a assistência nutricional auxilie de modo eficaz nas complicações relacionadas à doença, promova boas respostas ao tratamento, melhore o prognóstico e que proporcione uma melhor qualidade de vida a esses pacientes. Nesse sentido, o presente estudo teve por objetivo avaliar o perfil nutricional e alimentar de portadores de HIV-1 internados em um hospital universitário.

\section{MATERIAIS E MÉTODOS}

A pesquisa foi realizada na Clínica Doenças Infecciosas e Parasitárias (DIP), do Hospital Universitário João de Barros Barreto (HUJBB)/Universidade Federal do Pará (UFPA). Trata-se de um estudo transversal, descritivo. A população de estudo foi composta por 54 pacientes internados, de ambos os sexos, na faixa etária de 20 a 59 anos, acompanhados durante o período de Abril a Outubro de 2014, por demanda espontânea.

Para composição da casuística foram considerados os seguintes critérios: indivíduos que estavam conscientes e orientados; deambulando, sem edema; adultos de ambos os sexos e mulheres que não estavam gestantes.

Os pacientes foram submetidos à análise do perfil socioeconômico e demográfico, avaliação antropométrica e avaliação dietética. Para a realização do estudo foi utilizado formulário próprio para o levantamento das características demográficas (sexo e idade), socioeconômicas (estado civil, escolaridade e renda familiar), antropométricas (índice de massa corporal (IMC), prega cutânea tricipital (PCT), circunferência do braço $(\mathrm{CB})$ e circunferência muscular do braço $(\mathrm{CMB})$ ) e dietéticas (questionário de frequência alimentar (QFA)). As perguntas foram respondidas pelos próprios pacientes no momento da coleta de dados.

A avaliação antropométrica foi realizada mediante aferição das medidas de peso $(\mathrm{kg})$ e altura $(\mathrm{m})$, PCT, CB e CMB. O IMC foi obtido pela relação: peso atual (kg)/ altura $^{2}(\mathrm{~m})$ e foi classificado seguindo os parâmetros do World Health Organization $(\mathrm{WHO})^{7}$. O valor de PCT foi comparado com o padrão de referência de Frisancho ${ }^{8}$. Foi realizada a adequação, de acordo com a equação da adequação do percentual de prega cutânea tricipital (\%PCT). O estado nutricional da prega cutânea foi classificado através dos valores de referência, propostos por Blackburn \& Bistrian 9 . Para a medição da CB foi utilizada fita métrica inelástica, seguindo a técnica para mensuração, segundo Augusto ${ }^{10} \mathrm{e}$ Kamimura et al. ${ }^{11}$. O valor encontrado foi confrontado com os valores de referência estabelecido por Frisancho ${ }^{8}$, segundo os gêneros e idade. Os resultados foram classificados de acordo com os valores de referência propostos por Blackburn \& Thorntor $^{12}$. A CMB é utilizada para determinação da massa muscular ${ }^{11}$. Utilizam-se as medidas CB e PCT, através da fórmula. A sua interpretação realizou-se segundo padrão de normalidade de Frisancho ${ }^{8}$ e os resultados classificados 
através dos valores de referência adaptados de Blackburn \& Thorntor $^{12}$, citado por Kamimura et al. ${ }^{11}$.

Para identificação de hábitos alimentares foi realizado QFA adaptado de acordo com a regionalidade. Classificados em grupos: leite e derivados, energéticos (arroz, macarrão e farinha), proteínas (carne de gado, frango, peixe e ovos), reguladores, açúcares e ultraprocessados. O questionário apresentou quatro opções de frequência (diário, semanal, mensalmente e raramente ou nunca) e a análise foi feita segundo as recomendações da pirâmide alimentar e Guia Alimentar da População Brasileira ${ }^{13}$.

A análise dos dados ocorreu pela estatística descritiva, através das medidas de tendência central (média) e de medidas de variabilidade (desvio padrão), bem como a distribuição absoluta e relativa $(n-\%)$. Para verificar as diferenças de médias foi realizada a análise de variância (ANOVA) e o grau de associação entre as variáveis foi medido pela correlação de Pearson, em virtude da distribuição normal da amostra, estabeleceu-se como significância valor de $p<0,05$. Os dados da pesquisa foram compilados e armazenados em um banco de dados do programa Excel versão 2010 e posteriormente analisados no programa Bioestat 5.0 $0^{14}$.

A pesquisa foi aprovada pelo Comitê de Ética em Pesquisa em Seres Humanos do Hospital Universitário João de Barros Barreto, sob parecer no 637.225, de acordo com as normas da resolução no 466/12 e suas complementares do Conselho Nacional de Saúde/Ministério da Saúde do Brasil, e obteve-se a assinatura do Termo de Consentimento Livre e Esclarecido (TCLE), sendo assegurados a confiabilidade e o anonimato dos participantes.

\section{RESULTADOS}

Dos 54 pacientes que participaram do estudo, 53,7\% eram do sexo masculino. Referente à idade, houve predominância de 72,2\% entre 20 e 40 anos. Em relação à escolaridade, a maioria dos pacientes, 66,7\%, apresentou de 1 a 8 anos de estudo. A renda familiar de $88,9 \%$ dos pacientes estava entre 1 a 3 salários mínimos mensais e a maioria, $66,7 \%$, relatou não possuir companheiro(a).

Na Tabela 1 é apresentada a classificação do estado nutricional com valores médios e desvio padrão, segundo faixa etária. É possível observar em relação ao IMC que $(55,5 \%)$ apresentaram desnutrição com média e desvio-padrão de $(16,3 \pm 1,7)$ seguido de 38,9\% com eutrofia. Através da PCT observou-se que a maioria $(92,6 \%)$ apresentou diagnóstico de desnutrição com média e desvio-padrão de $(6,2 \pm 3,1)$. As variáveis $\mathrm{CB}$ e $\mathrm{CMB}$ também apresentaram predominância para desnutrição, 79,6 e 55,5\%, respectivamente.

O teste da correlação de Pearson (Tabela 2) demonstrou significativa correlação do IMC com as outras medidas antropométricas. O IMC e a PCT apresentaram-se moderada e positivamente correlacionadas $(p=0,014)$. Houve forte e positiva correlação entre o IMC e a CB $(p=0,0012)$, assim como entre o IMC e a CMB $(p=0,0007)$.

Tabela 1. Classificação das variáveis antropométricas segundo faixa etária em portadores de HIV/Aids.

\begin{tabular}{|c|c|c|c|}
\hline $\begin{array}{c}\text { Variáveis } \\
\text { Antropométricas }\end{array}$ & n (\%) & c (DP) & Anova \\
\hline \multicolumn{4}{|l|}{ IMC } \\
\hline Desnutrição & $30(55,5)$ & $16,3^{\mathrm{a}}( \pm 1,7)$ & \multirow{4}{*}{0,037} \\
\hline Eutrofia & $21(38,9)$ & $21,0^{\mathrm{b}}( \pm 1,9)$ & \\
\hline Sobrepeso & $2(3,7)$ & $27,0^{c}( \pm 0,8)$ & \\
\hline Obesidade & $1(1,9)$ & $37,0^{*}$ & \\
\hline \multicolumn{4}{|l|}{ РCT } \\
\hline Desnutrição & $50(92,6)$ & $6,2^{\mathrm{a}}( \pm 3,1)$ & \multirow{3}{*}{0,002} \\
\hline Eutrofia & $2(3,7)$ & $11,5^{\mathrm{b}}( \pm 2,1)$ & \\
\hline Obesidade & $2(3,7)$ & $17,0^{c}( \pm 1,4)$ & \\
\hline \multicolumn{4}{|l|}{$\mathrm{CB}$} \\
\hline Desnutrição & $43(79,6)$ & $22,2^{\mathrm{a}}( \pm 3,8)$ & \multirow{3}{*}{0,948} \\
\hline Eutrofia & $10(18,5)$ & $28,5^{\mathrm{a}}( \pm 2,1)$ & \\
\hline Obesidade & $1(1,9)$ & $43,0^{* *}$ & \\
\hline \multicolumn{4}{|l|}{$\mathrm{CMB}$} \\
\hline Desnutrição & $30(55,5)$ & $19,6^{\mathrm{a}}( \pm 3,7)$ & \multirow{3}{*}{0,282} \\
\hline Eutrofia & $18(33,4)$ & $23,27^{\mathrm{a}}( \pm 3,8)$ & \\
\hline Excesso de peso & $6(11,1)$ & $26,65^{\mathrm{a}}( \pm 5,6)$ & \\
\hline
\end{tabular}

IMC: Índice de massa corporal; PCT: Prega cutânea tricipital; CB: Circunferência do braço; CMB: Circunferência muscular do braço.

* Não há desvio padrão, pois apresenta somente um indivíduo.

abc Letras diferentes entre linhas representa diferença significativa entre médias pelo teste Anova.

Tabela 2. Correlação de Pearson entre variáveis antropométricas utilizadas na avaliação do estado nutricional de portadores de HIV/Aids.

\begin{tabular}{|c|c|c|}
\hline Variáveis & IMC & Valor de $p$ \\
\hline IMC & 1,00 & \\
\hline РCT & $0,545^{a}$ & 0,014 \\
\hline CB & $0,791^{b}$ & 0,0012 \\
\hline $\mathrm{CMB}$ & $0,632^{b}$ & 0,0007 \\
\hline
\end{tabular}

IMC: Índice de massa corporal; PCT: Prega cutânea tricipital. CB: Circunferência do braço. CMB: Circunferência muscular do braço.

${ }^{a}$ Correlação moderada; ${ }^{b}$ Correlação forte.

Analisando o questionário de frequência alimentar, de acordo com a frequência relativa da ingestão de alimentos energéticos, é observado consumo diário de arroz, pão e farinha e consumo semanal de batata, macarrão e biscoitos. A tapioca, nesta amostra pesquisada, se faz presente em sua maioria semanalmente. O consumo de balas, doces e chocolate é realizado raramente ou nunca, assim como para o azeite de oliva, manteiga ou margarina. Refrigerantes também estão nesta classificação de raramente ou nunca, conforme a Tabela 3.

Referente à frequência relativa da ingestão de alimentos construtores, observou-se que a amostra pesquisada, 
consome diariamente leite, referindo consumir raramente ou nunca queijos, as carnes (bovina, aves e peixes) apresentam um consumo semanal, assim como ovos. A maioria relatou consumir raramente ou nunca alimentos enlatados e embutidos. Em relação às leguminosas é feito consumo diário de feijão, no entanto a maioria dos pesquisados relataram consumir raramente ou nunca soja, conforme dados descritos na Tabela 4.
Com relação à frequência relativa da ingestão de alimentos reguladores, observou-se que o consumo de frutas é diário, ressaltando o consumo de açaí semanalmente. $\mathrm{O}$ consumo de frutas regionais (manga, cupuaçu, pupunha, tucumã, taperebá) em sua maioria é mensal. Em relação às hortaliças, estes pacientes mencionaram consumo diário de verduras e legumes. Raramente ou nunca consomem farelos (aveia, linhaça), valores estes referenciados na Tabela 5.

Tabela 3. Consumo de Alimentos Energéticos entre portadores de HIV/Aids.

\begin{tabular}{lcccc}
\hline $\begin{array}{c}\text { Alimentos Energéticos } \\
\text { Variável }(\mathbf{n = 5 4 )}\end{array}$ & $\begin{array}{c}\text { Diário } \\
\mathbf{n}(\%)\end{array}$ & $\begin{array}{c}\text { Semanal } \\
\mathbf{n}(\%)\end{array}$ & $\begin{array}{c}\text { Mensal } \\
\mathbf{n}(\%)\end{array}$ & $\begin{array}{c}\text { Raramente/Nunca } \\
\mathbf{n}(\%)\end{array}$ \\
\hline Manteiga & $22(41)$ & $5(9)$ & $0(0)$ & $27(50)$ \\
Margarina & $18(33)$ & $4(7)$ & $0(0)$ & $32(59)$ \\
Azeite de Oliva & $13(24)$ & $11(20)$ & $6(11)$ & $24(44)$ \\
Refrigerante & $10(19)$ & $18(33)$ & $2(4)$ & $24(44)$ \\
Doces* & $2(4)$ & $15(28)$ & $5(9)$ & $32(59)$ \\
Tapioca & $2(4)$ & $26(48)$ & $12(22)$ & $15(28)$ \\
Biscoito & $10(19)$ & $19(35)$ & $8(15)$ & $17(31)$ \\
Pão & $43(80)$ & $7(13)$ & $0(0)$ & $4(7)$ \\
Macarrão & $20(37)$ & $32(59)$ & $1(2)$ & $1(2)$ \\
Farinha & $30(56)$ & $10(19)$ & $1(2)$ & $13(24)$ \\
Batata Inglesa & $16(30)$ & $30(56)$ & $2(4)$ & $6(11)$ \\
Arroz & $32(59)$ & $16(30)$ & $2(4)$ & $4(7)$ \\
Total \% & 100 & 100 & 100 & 100 \\
\hline
\end{tabular}

* Açúcares.

Tabela 4. Consumo de Alimentos Construtores entre portadores de HIV/Aids.

\begin{tabular}{lcccc}
\hline $\begin{array}{c}\text { Alimentos Construtores } \\
\text { Variável (n=54) }\end{array}$ & $\begin{array}{c}\text { Diário } \\
\mathbf{n}(\%)\end{array}$ & $\begin{array}{c}\text { Semanal } \\
\mathbf{n}(\%)\end{array}$ & $\begin{array}{c}\text { Mensal } \\
\mathbf{n}(\%)\end{array}$ & $\begin{array}{c}\text { Raramente/Nunca } \\
\mathbf{n}(\%)\end{array}$ \\
\hline Leite & $39(72)$ & $8(15)$ & $1(2)$ & $6(11)$ \\
Queijo & $7(13)$ & $10(19)$ & $7(13)$ & $30(56)$ \\
Carne Bovina & $6(11)$ & $44(81)$ & $1(2)$ & $3(6)$ \\
Frango & $1(2)$ & $49(91)$ & $0(0)$ & $4(7)$ \\
Peixe & $2(4)$ & $45(83)$ & $3(6)$ & $4(7)$ \\
Ovo & $4(7)$ & $28(52)$ & $3(6)$ & $19(35)$ \\
Enlatados* & $1(2)$ & $18(33)$ & $5(9)$ & $30(56)$ \\
Embutidos* & $1(2)$ & $17(31)$ & $3(6)$ & $33(61)$ \\
Feijão & $32(59)$ & $21(39)$ & $1(2)$ & $0(0)$ \\
Soja & $0(0)$ & $3(6)$ & $2(4)$ & $49(91)$ \\
Total \% & 100 & 100 & 100 & 100 \\
\hline
\end{tabular}

* Ultraprocessados.

Tabela 5. Consumo de Alimentos Reguladores entre portadores de HIV/Aids.

\begin{tabular}{lcccc}
\hline $\begin{array}{c}\text { Alimentos Reguladores } \\
\text { Variável (n=54) }\end{array}$ & $\begin{array}{c}\text { Diário } \\
\mathbf{n}(\%)\end{array}$ & $\begin{array}{c}\text { Semanal } \\
\mathbf{n}(\%)\end{array}$ & $\begin{array}{c}\text { Mensal } \\
\mathbf{n}(\%)\end{array}$ & $\begin{array}{c}\text { Raramente/Nunca } \\
\mathbf{n}(\%)\end{array}$ \\
\hline Frutas regionais & $6(11)$ & $13(24)$ & $20(37)$ & $15(28)$ \\
Outras frutas & $27(50)$ & $22(41)$ & $1(2)$ & $4(7)$ \\
Açaí & $11(20)$ & $29(54)$ & $4(7)$ & $10(19)$ \\
Legumes & $24(44)$ & $18(33)$ & $4(7)$ & $8(15)$ \\
Verduras & $24(44)$ & $18(33)$ & $3(6)$ & $2(17)$ \\
Farelos* & $9(17)$ & $20(37)$ & 100 & 100 \\
Total \% & 100 & 100 & & $141)$ \\
\hline
\end{tabular}

* Aveia, linhaça. 


\section{DISCUSSÃO}

Dos 54 pacientes investigados, a maioria foi do sexo masculino, característica semelhante descrita no estudo proposto por Grangeiro et al. ${ }^{15}(85,6 \%)$ e Pieri et al. ${ }^{16}$ (62\%). Ainda observa-se predominância de casos da infecção entre homens em relação às mulheres, o que se fundamenta no fato do homem preocupar-se pouco com sua saúde, principalmente no que diz respeito à prevenção de doenças. No entanto, nota-se questões relacionadas à feminização da AIDS caracterizadas, geralmente, pela ideia de invulnerabilidade das mulheres, por viverem em um relacionamento estável e pelas dificuldades que elas encontram ao negociar o uso do preservativo com o parceiro ${ }^{17}$.

A maior incidência de pacientes com a doença na faixa etária de 20 a 40 anos também foi observado por Motta et al. $^{18}$. A grande incidência nessa faixa etária pode ser justificada por compreender um período de maior atividade sexual, associado a um maior descuido no modo de prevenção, como o uso de preservativos e consequentemente a falta deste compromisso levar a uma maior capacidade para se propagar a doença ${ }^{18}$.

Acerca do perfil socioeconômico demonstra-se que os pacientes pertencem a um nível econômico baixo, apresentando também uma baixa escolaridade. Esses resultados são semelhantes ao achado obtido por Motta et al. ${ }^{18}$, que observou que o número de pessoas infectadas pelo HIV com baixo padrão socioeconômico está aumentando. Observa-se que a maioria dos pacientes relatou não possuir companheiro, corroborando os dados de Padoin et al. ${ }^{17}$. O baixo nível de escolaridade detectado torna-se relevante, pois a compreensão sobre a enfermidade requer um nível satisfatório de escolaridade. Uma possível explicação é que populações com mais anos de estudo têm maior acesso à informação, métodos de prevenção e compreensão do impacto positivo do tratamento na evolução clínica da doença. A infecção do HIV/AIDS mostra-se intimamente ligada aos aspectos mencionados: baixa escolaridade e classes econômicas menos favorecidas ${ }^{16}$.

De acordo com o IMC, revelou-se diagnóstico de desnutrição. Estudo semelhante realizado por Ribeiro ${ }^{19}$ observou elevada predominância de baixo peso em pacientes hospitalizados por HIV/AIDS. Resultados controversos foram encontrados por Silva et al..$^{20}$, no qual a grande maioria dos pacientes foi classificada como eutróficos. Uma justificativa para essa discordância é que em pacientes internados observa-se uma maior gravidade em seu estado clínico, assim o estado nutricional desses pacientes é mais comprometido do que dos pacientes não hospitalizados ${ }^{19}$.
A desnutrição, uma das complicações da AIDS, é de grande significância para o prognóstico da evolução da doença, pois compromete a função imunológica, causa danos ao metabolismo, diminui a resposta às terapias e causa progressiva debilidade, levando a uma diminuição da sobrevida e ao comprometimento da qualidade de vida dos pacientes ${ }^{21}$.

No presente estudo, o uso da PCT demonstrou predominância para desnutrição, corroborando com os achados de Ladeira \& Silva ${ }^{22}$, que mostraram que a maioria dos pacientes apresentou uma baixa reserva de gordura subcutânea. O uso da CB evidenciou ocorrência de desnutrição, semelhante ao encontrado por Feitosa \& Cabral ${ }^{23}$, que acharam valores altos desse parâmetro. Segundo Guterres $^{24}$, a CB representa a soma das áreas constituídas pelos tecidos gorduroso, muscular e ósseo, portanto, valores baixos de $\mathrm{CB}$ relacionam-se a um déficit nutricional geral. Quanto a $\mathrm{CMB}$, o diagnóstico referese também a desnutrição. Foram encontrados resultados contrários nos estudos de Gomes \& Lourival $^{25}$, no qual houve prevalência de eutrofia indicando adequada reserva de massa muscular.

A perda de massa magra, considerada a massa celular, está vinculada a um pior prognóstico da doença ${ }^{26}$. De acordo com Moutinho et al. ${ }^{21}$, diversos mecanismos podem estar relacionados à ocorrência de desnutrição em pacientes com AIDS, tais como: baixa ingestão calórico-proteica, alterações metabólicas, diarreias, infecções oportunistas, alterações neurológicas, fatores psicológicos, interação droga-nutriente e deficiência de vitaminas A, C, B12, B6 e minerais como zinco e selênio.

No presente estudo, observou-se correlação significativa entre as variáveis antropométricas. O IMC correlaciona-se em grande magnitude com as demais variáveis (PCT, CB e $C M B$ ). Constata-se que houve uma moderada correlação entre o IMC e a PCT e forte correlação entre IMC com a CB e $\mathrm{CMB}$, ou seja, quando o paciente apresentava seu estado nutricional classificado em desnutrição pelo IMC, também seu estado nutricional se deteriorava em relação às medidas da $\mathrm{CB}$ e da $\mathrm{CMB}$, correlação diretamente proporcional e positiva. Visto isso, a utilização do IMC deve ser combinada com demais medidas para se avaliar de modo mais eficaz e minucioso o estado nutricional do paciente. O IMC associado às variáveis utilizadas no presente estudo possibilitou obterse uma ideia mais precisa da composição e distribuição de massa magra e gordura corporal.

Analisando a ingestão dietética, observou-se que no grupo dos alimentos energéticos, o consumo de cereais, massas, tubérculos é bastante expressivo, ressaltando o consumo diário de alimentos como arroz, farinha e pão. É frequente 
o consumo de massas e biscoitos entre a amostra. Verificouse baixa frequência de consumo de alimentos gordurosos e industrializados, para a maioria dos pacientes do estudo, demonstrando ser um resultado satisfatório, mesmo o maior número não recebendo orientações de um profissional adequado sobre reduzir esses alimentos antes de serem hospitalizados. No entanto, demonstraram conhecimentos básicos do que seria uma alimentação saudável.

É de suma importância um controle no consumo de alimentos gordurosos, visto que a alteração no metabolismo dos lipídios é frequente em pacientes que vivem com HIV, independente do uso da Terapia Antirretroviral de Alta Potência (TARV), pois somente a infecção pelo vírus já é capaz de causar dislipidemias em 50\% dos casos, principalmente pelo HDL baixo ${ }^{27}$.

Observou-se que o grupo dos alimentos construtores, apresentou consumo diário de leite, sendo relevante também o consumo diário de feijão. Dentre as carnes, o frango apresentou o maior consumo semanal, seguido de peixe. Segundo o Guia Alimentar da População Brasileira ${ }^{13}$, as tendências nacionais de consumo desses grupos de alimentos, especialmente das carnes, são crescentes. Isso pode se justificar pelo fato de que o menor poder aquisitivo desses indivíduos não possibilita uma grande aquisição de alimentos, levando-os a ter uma alimentação mais básica e tradicional, que por sua vez apresenta itens de boa qualidade nutricional, a exemplo do arroz e feijão. Pessoas com maior renda costumam se alimentar fora de casa e tendem a consumir alimentos mais gordurosos, como refrigerantes, frituras e fast food.

Em relação aos alimentos reguladores referidos no estudo, como as frutas, verduras e legumes, os mesmos apresentam um consumo diário, no entanto, a ingestão ainda é pequena quando comparado ao consumo recomendado pelo Guia Alimentar da População Brasileira ${ }^{13}$. Evidencia-se o baixo consumo de frutas regionais, com exceção do açaí e alimentos protetores como farelos.

A Pesquisa de Orçamento Familiar ${ }^{28}$, realizada em 2008 e 2009, apontou que o arroz, feijão, carne bovina e pão apresentaram maiores frequências de consumo. Na Região Norte destaca-se o consumo de farinha de mandioca, peixes frescos e de açaí, mostrando similaridade com os resultados encontrados nesse estudo, onde o consumo desses alimentos foi significativo dentre a amostra. Segundo Coura et al. ${ }^{29}$ para se ter uma alimentação saudável, é preciso consumir uma variedade de alimentos em quantidade adequada, como frutas, verduras e legumes, diminuindo o consumo de doces, frituras e gorduras saturadas.

Quanto à limitação do estudo, em função da maior gravidade do quadro clínico de alguns pacientes, não foi possível realizar a avaliação nutricional completa, ocasionando no pequeno número da amostra.

Os resultados deste estudo mostraram que a maioria dos pacientes apresentou estado nutricional de desnutrição, com perda de massa magra e gordura corporal. Ressaltase a utilização do IMC combinado com outras variáveis antropométricas para um diagnóstico mais eficaz do estado nutricional desses indivíduos. O perfil alimentar, de modo geral, se caracterizou por prevalência de hábitos alimentares saudáveis dos mesmos. Sugere-se que novos estudos sejam conduzidos com a finalidade de avaliar o estado nutricional e a qualidade da alimentação de pacientes internados por HIV/AIDS no Brasil, considerando que essas informações são essenciais para guiar a execução do diagnóstico precoce e também servir como base para a terapêutica nutricional, subsidiando proporcionar estratégias nutricionais que venham auxiliar na potencialização da melhora clínica e nutricional de pacientes com HIV.

\section{REFERÊNCIAS}

1. Santos FF, Pereira FB, Silva CLO, Lazzarotto AR, Petersen RDS. Características imunológicas e virológicas e as variáveis flexibilidade (FLEX) e força de resistência abdominal (FRA) de crianças e adolescentes portadores de HIV/AIDS em uso de TARV. Rev Bras Med Esporte. 2014;19(1):40-43. https://doi.org/10.1590/S151786922013000100008

2. Perrut JF, Santos EP, Oliveira BR, Carneiro ACLL, Oliveira GL, Ervilha Junior, AB, Pacheco RC. Risco nutricional e sua associação com o tempo e desfecho da internação de pacientes com AIDS em um hospital de referência em infectologia de Belo Horizonte. Espaç Saúde. 2014;5(1):57-65.

3. Martins TA, Kerr LRFS, Kendall C, Mota RMS. Cenário Epidemiológico da Infecção pelo HIV e AIDS no Mundo. Rev Fisioter S Fun. 2014;3(1):4-7.

4. Silva, AMTM. VIH e nutrição: o combate a doença através da intervenção nutricional [trabalho acadêmico]. Porto: Universidade Fernando Pessoa; 2013.

5. Sena EA, Freitas CHSM, Pontes ALS. O Papel do Nutricionista na Atenção aos Portadores do HIV/AIDS no Sistema Penitenciário Brasileiro: uma Revisão da Literatura. Rev Bras Ciên Saúde. 2014;18(2):169-78. https://doi.org/10.4034/RBCS.2014.18.s2.12

6. Bassichetto KC, Bergamaschi DP, Garcia VRS, Veras MASM. Fatores associados à desnutrição em pessoas com 20 anos e mais, com HIV/ AIDS, em serviços públicos de saúde no Município de São Paulo, Brasil. Cad Saúde Pública. 2014;30(12):1-9.

7. Wold Health Organization. Physical status: the use and interpretation of anthropometry. Geneva: WHO; 1995.

8. Frisancho AR. New norms of upper limb fat and muscle áreas for assessent of nutritional status. J Clin Nutri. 1981;34(1):2540-5.

9. Blackburn LL, Bistrian BR. Nutritional and melabolic assessment of the hospitalized patient. JPEN. 1977;1(1):11-22. https://doi. org/10.1177/014860717700100101

10. Augusto ALP, Alves DC, Mannarino IC. Terapia nutricional. São Paulo: Atheneu; 1995. 
11. Kamimura MA, Baxman A, Sampaio LR, Cuppari L. Avaliação nutricional. In: Cuppari L. Nutrição clínica do adulto. $2^{\underline{a}}$ ed. Barueri: Manole; 2005. p. 89-127.

12. Blackburn GL, Thornton PA. Nutritional assessment of the hospitalized patient. Med Clin North Am. 1979;63(5):11103-15. https://doi.org/10.1016/S0025-7125(16)31663-7

13. Guia Alimentar para a população brasileira: promovendo a alimentação saudável. Edição especial. Brasília: Ministério da Saúde; 2006

14. Ayres M, Ayres Junior M, Ayres DL, Santos AS. BioEstat 5.0: aplicações estatísticas nas áreas das ciências biológicas e médicas. Belém: MCT; 2007.

15. Grangeiro A, Holcman MM, Onaga ET, de Alencar HDR, Placco ALN, Teixeira PR. Prevalência e vulnerabilidade a infecção pelo HIV de moradores de rua em São Paulo, SP. Rev Saúde Pública. 2012;46(4):674-84. https://doi.org/10.1590/S003489102012005000037

16. Pieri FM, Laurenti R. HIV/AIDS: perfil epidemiológico de adultos internados em Hospital Universitário. Cienc Cuid Saúde. 2012; 11:144-52. https://doi.org/10.4025/cienccuidsaude.v11i5.17069

17. Padoin SMM, Zuge SS, Santos EEP, Primeira MR, Aldrighi JD, Paula CC. Adesão a terapia antirretroviral para HIV/AIDS. Cogitare Enferm. 2013;18(3):446-51. https://doi.org/10.5380/ce.v18i3.33553

18. Motta WKS, Nóbrega DRM, Santos MGC, Gomes DQC, Godoy GP, Pereira JV. Aspectos demográficos e manifestações clínicas bucais de pacientes soropositivos para o HUV/Aids. Rev Odontol UNESP. 2014;43(1):61-7. https://doi.org/10.1590/S180725772014000100010

19. Ribeiro CSA. Prevalência de alterações nutricionais e fatores de risco para desnutrição em pacientes hospitalizados por HIV/AIDS [dissertação]. Salvador: Universidade Federal da Bahia; 2010.

20. Silva VS, Mori RMSC, Guimarães SM. Alterações Nutricionais em Pacientes com Lipodistrofia Associada ao HIV/Aids de uma Unidade de Referência do Município de Belém - Pará. DST - J Bras Doenças
Sex Transm. 2012;24(4):233-8. https://doi.org/10.5533/DST-21778264-201224403

21. Moutinho ABA, Pretto ADB, Moreira NA. Evolução do estudado nutricional de pacientes com AIDS atendidos em um ambulatório de nutrição. RBONE. 2015;9(51):85-95.

22. Ladeira POC, Silva DCG. Estado nutricional e perfil alimentar de pacientes assistidos pelo Programa de DST/AIDS e Hepatite Virais de um Centro de Saúde de Itaperuna-RJ. DST - J Bras Doenças Sex Transm. 2012;24(1):28-31. https://doi.org/10.5533/2177-8264201224108

23. Feitosa SMC, Cabral CP. Anemia em pacientes HIV-positivo atendidos em um Hopistal universitário de Pernambuco-Nordeste do Brasil. DST - J Bras Doenças Sex Transm. 2011;23(2):69-75. https://doi.org/10.5533/2177-8264-201123204

24. Guterres AS. Implantação de um protocolo ambulatorial de acompanhamento, orientação e terapia nutricional a portadores do HIV-1 [tese]. Belém: Universidade Federal do Pará; 2014.

25. Gomes TB, Lourival NBS. Perfil nutricional de pacientes HIV positivo do município de Apucarana (PR). SaudPesq. 2016;9(1):83-92. https://doi.org/10.17765/1983-1870.2016v9n1p83-92

26. Santos KM. Intervenções neurológicas em pacientes adultos infectados pelo Vírus da Imunodeficiência Humana. Int J Nutrol. 2016;9(1):119-27.

27. Da Silva IRP, Dias RM, Dutra CDT, Mendes ANL, Libonati RMF. Dislipidemia e estado nutricional em pacientes HIV positivo com síndrome lipodistrófica. Rev Epidemiol Control Infect. 2014;4(3):200-7. https://doi.org/10.17058/reci.v4i3.4878

28. IBGE. Instituto Brasileiro de Geografia e Estatística. Pesquisa de orçamentos familiares: 2008-2009. Rio de Janeiro: IBGE; 2011 [citado em 2015 ago. 23]. Disponível em: http://biblioteca.ibge. gov.br/visualizacao/livros/liv50063.pdf

29. Coura CF, Guerra VR, Nery LCL. Lipodistrofia em crianças infectadas com HIV sob terapia anti-retroviral: o desafio do século XXI. Pediatria. 2011;33(11):45-51. 